\title{
Correction of acknowledgments: PD-L1 testing in non-small cell lung cancer: past, present, and future
}

\author{
Hyojin Kim ${ }^{1}$, Jin-Haeng Chung ${ }^{1,2}$ \\ 1Department of Pathology, Seoul National University Bundang Hospital, Seongnam; \\ 2Department of Pathology, Seoul National University College of Medicine, Seoul, Korea
}

To the Editor:

We found an error in our published article.

Kim H, Chung JH. PD-L1 testing in non-small cell lung cancer: past, present, and future. Journal of Pathology and Translational Medicine 2019; 53(4): 199-206. https://doi.org/10.4132/jptm.2019.04.24.

On page 204, the Acknowledgments should be corrected as follows: This work was supported by the National Research Foundation of Korea (NRF) Grant funded by the Korean Government (MSIT) (No. 2017R1A5A1015626) and the Korea Health Technology R\&D Project through the Korea Health Industry Development Institute (KHIDI), funded by the Ministry of Health \& Welfare, Republic of Korea (grant number: HI17C1290).

We apologize for the error. 